\title{
Development of Petroleum-Based Carbon Composite Materials Containing Graphite/silicon Particles and Their Application to Lithium Ion Battery Anodes
}

\author{
Soon Young Noh, Younghoon Kim, Chul Wee Lee, and Songhun Yoon* \\ Green Chemistry Division, Korea Research Institute of Chemical Technology (KRICT), Daejeon 305-600, Korea
}

\begin{abstract}
:
Herein, a novel preparation method of highly homogeneous carbon-silicon composite materials was presented. In contrast to conventional solvent evaporation method, a milled silicon-graphite or its oxidized material were directly reacted with petroleum-derived pitch precursor. After thermal reaction under high pressure, pitch-graphite-silicon composite was prepared. Carbon-graphite-silicon composite were prepared by an air-oxidization and following carbonization. From energy dispersive spectroscopy, it was observed that small Si particles were highly embedded within carbon, which was confirmed by disappearance of Si peaks in Raman spectra. Furthermore, X-ray diffraction and Raman spectra revealed that carbon crystallinity decreased when the strongly oxidized silicon-graphite was added, which was probably due to oxygen-induced cross-linking. From the anode application in lithium ion batteries, carbon-graphite-silicon composite anode displayed a high capacity $\left(565 \mathrm{mAh} \mathrm{g}^{-1}\right)$, a good initial efficiency (68\%) and an good cyclability ( $88 \%$ retention at 50 cycles), which were attributed to the high dispersion of Si particles within cabon. In case of the strongly oxidized silicongraphite addtion, a decrease of reversible capacity was observed due to its low crystallinity.
\end{abstract}

Keywords : Pitch-graphite-Si composite, Petroleum pitch, Anode in lithium ion batteries

Received May 30, 2011 : Accepted June 6, 2011

\section{Introduction}

Carbon-based materials have been widely applied to the anodes of conventional lithium ion batteries. Because of low material cost and high rate performance, carbon materials prepared at low temperature $\left(<1500^{\circ} \mathrm{C}\right)$ have been currently considered as anode candidate in the batteries for HEV (hybrid electric vehicle) and plugin HEV. Especially, these carbons satisfy the important performance requirements such as a pulse power capability and a sloped-voltage profile for easy state of charge (SOC) prediction. ${ }^{1)}$ In order to improve their low capacity problem (250 400 $\mathrm{mAh} \mathrm{g}^{-1}$ ), however, composite preparation with a high capacity silicon (theoretical capacity

'Corresponding author. Tel.: +82-42-860-7199

E-mail address: yoonshun@krict.re.kr is $\left.3580 \mathrm{mAh} \mathrm{g}^{-1}\left(\mathrm{Li}_{15} \mathrm{Si}_{4}\right)\right)$, has been investigated. ${ }^{2-8)}$ Especially, the nanosized Si composite with carbon have exhibited an improved anode performance. ${ }^{5-8)}$ However, complicate preparation and high price of the nanosized Si can be shortcomings in its practical application. For the purpose of practical preparaton, the solvent evaporation method has been employed for pitch-graphite-silicon composite. In this method, the pitch as carbon precursor was dissolved in solvent and Si metal was added into the pitch solution, followed by solvent evaporation and carbonization. ${ }^{2-4)}$ The pitch is usually prepared by thermal reaction under high pressure using hydrocarbon precursors from petroleum or coaltar. $^{9-11)}$ In the pitch-graphite-silicon composite preparation, the pitch is dissolved in organic solvent and converted into carbonaceous content with thermally inert $\mathrm{Si}$. When this material was applied to anode in lithium ion batteries, 
a higher capacity than carbon and a better cycleability than Si have been observed. ${ }^{3)}$ A delicate control of evaporating solvent is, however, required in order to avoid a separation between the pitch and Si particles, which gave rise to the addtion of excess amount $\mathrm{Si}$ and the poor cycle performance. ${ }^{2-4)}$

In order to overcome these problems, herein, a novel preparation of carbon-graphite-silicon composite is developed. A milled graphite-Si powder is directly reacted with a petroleum-derived pitch precursor, which requires no solvent evaporation process. In order to investigate an oxidation effect in the pitch formation, a strong acid treatment is also conducted for the milled graphite-Si powder. After thermal reaction and following carbonization, carbon-graphite-silicon composite materials are obtained. The structural and morphological analysis are carred out using scanning electron microscope (SEM), X-ray diffraction (XRD) and Raman spectroscopy. Anode performance in lithium ion batteries is investigated using electrodes fabricated by the prepared composite materials.

\section{Experimental}

\subsection{Preparation of composite materials}

Graphite and Si powder were purchased from Aldrich company. They were mixed with the weight ratio of $10: 1$. Graphite and Si power was milled using planetery mill at $300 \mathrm{rpm}$ for $3 \mathrm{hrs}$. The miled graphite-Si powder was mixed with a solution of strong acids composed of $\mathrm{HNO}_{3} / \mathrm{H}_{2} \mathrm{SO}_{4} / \mathrm{HClO}_{4}$. (The weight ratio : graphite/ $\left.\mathrm{HNO}_{3} / \mathrm{H}_{2} \mathrm{SO}_{4} / \mathrm{HClO}_{4}=18 / 10 / 56 / 16\right)$ was added into at $135^{\circ} \mathrm{C}$ for $7 \mathrm{hr}$. After this reaction, $1 \mathrm{~g}$ oxidation agent $\mathrm{V}_{2} \mathrm{O}_{5}$ was added for $2 \mathrm{hr}^{12)}$ After filtration and washing using water, an oxidized graphite-Si composite was obtained. As a pitch precursor, pyrolyzed fuel oil (PFO) was provided by EiL Chemical Co. GC-mass analysis using $\mathrm{HP}^{\circledR}$ was conducted in order to identify the components in PFO. The milled graphite/Si powder or its oxidized material were added into the pitch preparation reaction, which was conducted using $1 \mathrm{~L}$ batch type reactor at $300^{\circ} \mathrm{C}$ under $10 \mathrm{~atm}$. Typical $\mathrm{PFO} /$ graphite/ $\mathrm{Si}$ weight ratio was 90/9/1. After crushing pitch composite, the air-oxidation treatment was conducted at $200^{\circ} \mathrm{C}$ and the carbonization was carried out at 750,850 and $1000^{\circ} \mathrm{C}$ for $4 \mathrm{hr}$.

\subsection{Structural and electrochemical analysis} JEOL JSM-840A SEM apparatus was utilized for morphological and compositional analysis. X-ray diffraction (XRD) patterns were obtained with a Rigaku D/Max$3 \mathrm{C}$ diffractometer equipped with rotating anode and $\mathrm{Cu} \mathrm{K} \alpha$ radiation $(\lambda=0.15418 \mathrm{~nm})$. Raman spectra were recorded using the $532 \mathrm{~nm}$ line from a laser of $20 \mathrm{~mW}$. IR spectra were obtained using $\mathrm{KBr}$ pellet in Bruker FT-IR spectrometer.

To prepare the anode, prepared pitch-graphite-silicon composite powder/conducting agent (Super P)/PVDF (polyvinylidenfluoride) binder with a weight ratio of 8/1/1. Electrode fabrication method and coin cell analysis were identical to that reported in the literature, except for the voltage range from 2.5 to $0.001 \mathrm{~V} v s$. $\mathrm{Li} / \mathrm{Li}^{+}{ }^{+13,14)} \mathrm{The}$ applied current (rate) and electrode loading were $15 \mathrm{~mA} \mathrm{~g}^{-1}$ and $15 \mathrm{mg} \mathrm{cm}^{-2}$, respectively. Cycling performance was analyzed using WBCS3000 Wonatech ${ }^{\circledR}$ company and ac-impedance analysis was carried out using Iviuumstat from Iviuum ${ }^{\circledR}$ company.

\section{Results and Discussion}

\subsection{Composite preparation and analysis}

Fig. 1(a) and (b), show particle morphology of pristine graphite and Si powder before milling, respectively. Particle size of graphite and Si was about 10 20 $\mu \mathrm{m}$. After mixing graphite and Si power in the weight ratio of $10: 1$, milling was carried out for 3 hours using planetary mill. Fig. 1(c) displays a milled graphite/Si powder. As seen, overall particle size was below $10 \mu \mathrm{m}$ and homogeneous morphology was observed. This milled graphite and silicon, denoted as mGS, was added to a batch reactor for pitch formation. From the literatures, it has been reported that the addition of strong oxidants such as $\mathrm{HF}$ and $\mathrm{BF}_{3}$ increased pitch yield. ${ }^{17}$ ) Along this line, a similar effect can be expected by an increase of oxygen-containing surface functional groups in carbon material added into pitch formation reaction. A strong oxidation was carried out by addition of $\mathrm{mGS}$ into the solution of strong acids composed of $\mathrm{HNO}_{3}$, $\mathrm{H}_{2} \mathrm{SO}_{4}$ and $\mathrm{HClO}_{4}$ at $135^{\circ} \mathrm{C}$ for $7 \mathrm{hr}$, followed by vanadium oxide addition. ${ }^{8)}$ After filtration and washing, an oxidized graphite-Si composite (O-mGS) was obtained. EDS analysis displayed that $\mathrm{mGS}$ and $\mathrm{O}-\mathrm{mGS}$ contained $23 \%$ and $43 \%$ oxygen, respectively, indicative of large increase of oxygen functionality in O-mGS. Also, FT-IR analysis was conducted to identify the surface functional groups. In Fig. 2(a), change of IR spectra by acid treatment is displayed in the $4000-400 \mathrm{~cm}^{-1}$ region. As seen, mGS and O-mGS showed a strong and broad peak 


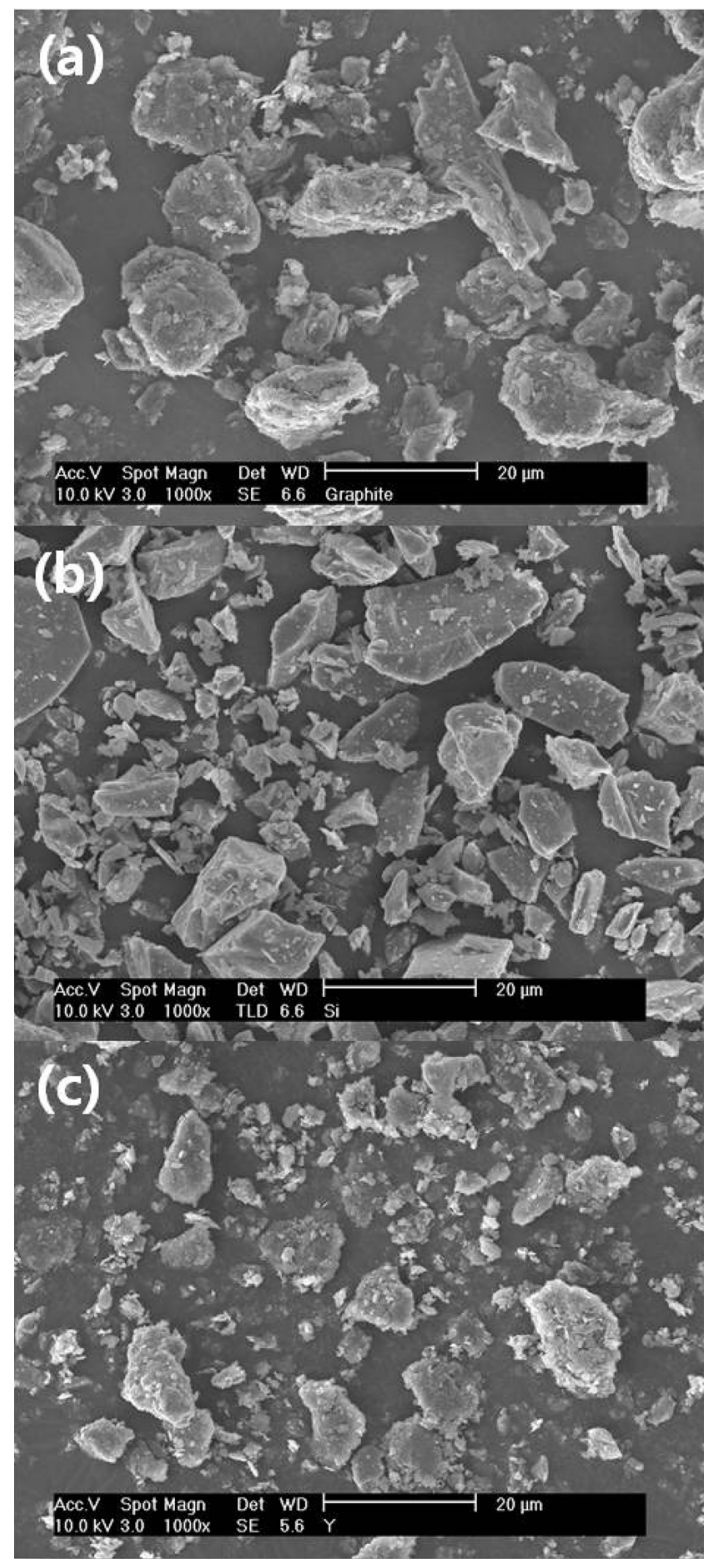

Fig. 1. SEM images of (a); pristine graphite, (b); pristine $\mathrm{Si}$ powder and (c); milled graphite-Si powder.

near $3434 \mathrm{~cm}^{-1}$, corresponding to $\mathrm{O}-\mathrm{H}$ and $\mathrm{N}-\mathrm{H}$ stretching in multiple hydrogen-bonded compounds. ${ }^{15)}$ Note that ball milling can possible lead to some surface oxidation of mGS. Particularly, peaks associated with oxygen and sulfur containing functional groups became much higher in O-mGS, which were located between $1000 \mathrm{~cm}^{-1}$ and $1300 \mathrm{~cm}^{-1}$; the $-\mathrm{C}-\mathrm{O}$ vibration in alkyl-aryl ether and $\mathrm{S}=\mathrm{O}$ stretching. ${ }^{16)}$

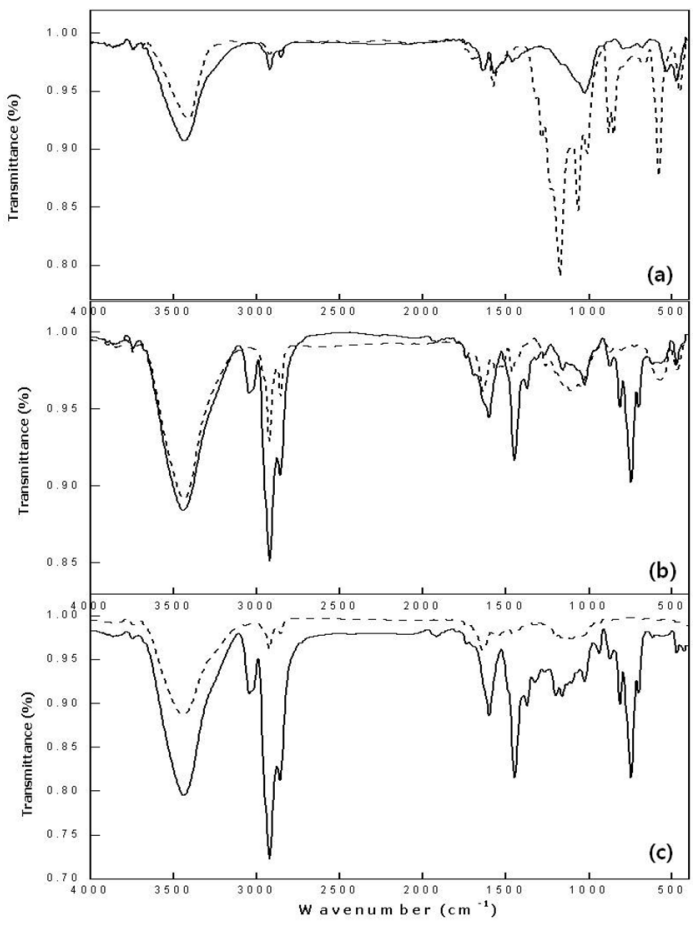

Fig. 2. FT-IR spectra of (a); milled Si-graphite (solid line) and its chemically oxidized material (dash line), (b); 10-mGS pitch (solid line) and 10-mGS-750 carbon (dash line), (c); 10-O-mGS pitch (solid line) and 10-O-mGS-750 carbon (dash line).

Using the mGS and O-mGS, the pitch formation reaction was conducted. Here, PFO was used as a main pitch source, which was produced by naphtha cracking process in petroleum industry. Since PFO is very cheap and easy to obtain in a large mass, it can be a suitable candidate for anode carbon precursor in lithium ion batteries. From our preliminary GC-mass analysis, it was shown that PFO were composed of $17 \mathrm{wt} \%$ naphthalene, $12 \mathrm{wt} \%$ methyl naphthalene, $12 \mathrm{wt} \%$ benzene derivatives, $13 \mathrm{wt} \%$ indene and the other smaller aromatic molecules. These aromatic hydrocarbons can be used for the preparation of petroleumbased pitch. ${ }^{17)}$ Hence, we employed PFO as a precursor of anode carbon of lithium ion batteries. After mixing PFO with the mGS or O-mGS in the weight ratio of $10: 1$, a thermal treatment at $300^{\circ} \mathrm{C}$ was conducted under $10 \mathrm{~atm}$ pressure in order to obtain pitch/graphite/Si composite materials. During this reaction, thermal condensation reaction between PFO molecules via. radical polymerization proceeded and a high molecular weight materials of PAHs(Polycyclic aromatic hydrocarbons) were 
obtained as a pitch form. ${ }^{11)}$ The softening point of as-prepared pitch-graphite-Si composite was about $100^{\circ} \mathrm{C} .^{9,11)}$ This composite was crushed into smaller particles and air-oxidized at $200^{\circ} \mathrm{C}$ using air blowing in order to thermoset the composite pitch. During this oxidation, the bloating of pitch can be prevented, which can give rise to the phase separation between Si-graphite and pitch during carbonization. ${ }^{9)}$ After oxidation, the obtained materials were carbonized at several temperatures (750, $850,1000^{\circ} \mathrm{C}$ ) for $4 \mathrm{hr}$. During this process, the oxidized pitch-graphite-Si composite material was converted into carbonaceous composite materials. We named the final composite materials as x-mGS-y and x-O-mGS-y, which were acquired by the addition of $\mathrm{mGS}$ and $\mathrm{O}-\mathrm{mGS}$, respectively. Here, $\mathrm{x}$ and $\mathrm{y}$ are denoted as the added amount $(\%)$ of graphite-Si and the carbonization temperature $\left({ }^{\circ} \mathrm{C}\right)$, respectively.

From Table 1, pitch and carbonization yields of 0 mGS, 10-mGS and 10-O-mGS are listed according to the carbonization temperatures. The pitch yields of 10-mGS and $10-\mathrm{O}-\mathrm{mGS}$ are 27.4 and $30.2 \%$, respectively. The observed increase of pitch yield is probablely due to the higher cross-linking attributed by large amount of oxygencontaining functional groups in $10-\mathrm{O}-\mathrm{mGS}$ case. ${ }^{17,18)}$ This implies that the pitch yield can increase without the addition of very toxic and dangerous chemicals such as $\mathrm{HF}$ and $\mathrm{BF}_{3}{ }^{17)}$ After carbonization of $0-\mathrm{mGS}$ and 10 -mGS, the yield decreased at $850^{\circ} \mathrm{C}$ and its slight increase was observed after $1000^{\circ} \mathrm{C}$ carbonization, indicative of a possible retarded carbon gasification in the latter case. ${ }^{19)}$ Note that the carbonization yield of 10-O-mGS remained invariant irrespective of carbonization temperatures, which was probably attributed to the high-cross linking between molecules.

In order to investigate chemical structure of as-prepared composite pitches and their carbonized products, FT-IR analysis was carried out and its results are displayed in Fig. 2(b) and (c). As seen, strong absorbance was observed at $2924,2855,1458$ and $1376 \mathrm{~cm}^{-1}$ corresponded to the

Table 1. Yield of pitch formation and carbonization yields according to the temperature

\begin{tabular}{ccccc}
\hline & Pitch yield $\% \%$ & \multicolumn{3}{c}{ Carbonization yield } \\
\cline { 3 - 5 } & & at $750^{\circ} \mathrm{C} \%$ & at $850^{\circ} \mathrm{C} \%$ & at $1000^{\circ} \mathrm{C} \%$ \\
\hline 0 - mGS & 25.5 & 42.4 & 20.0 & 28.8 \\
$10-\mathrm{mGS}$ & 27.4 & 57.3 & 30.0 & 38.6 \\
$10-\mathrm{O}-\mathrm{mGS}$ & 30.2 & 41.7 & 40.8 & 41.6 \\
\hline
\end{tabular}

distribution of aliphatic hydrogen between the $-\mathrm{CH}_{2}$ and $-\mathrm{CH}_{3}$ structures caused by $\mathrm{C}-\mathrm{H}$ stretching in $-\mathrm{CH}_{3}$ and $-\mathrm{CH}_{2}$ in pitch materials. The presence of peaks at $1601,1029,812$ and $747 \mathrm{~cm}^{-1}$ was observed in pitches, which was attributed to $-\mathrm{CH}_{3}$ substituents on the aromatics rings in all fractions. ${ }^{15)}$ The spectral band at $747 \mathrm{~cm}^{-1}$ was attributed to the four adjacent hydrogen atoms on the aromatic ring and the band at $812 \mathrm{~cm}^{-1}$ to two or three adjacent hydrogen atoms. After carbonization, the intensity of the observed peaks was largely decreased but broad peak near $3500 \mathrm{~cm}^{-1}$ remained, indicating that the heat treatment temperature of $750^{\circ} \mathrm{C}$ was too low to remove all oxygen and nitrogen containing functional groups. ${ }^{10)}$ Furthermore, note that the chemical treated 10-O-mGS750 material showed a more drastic decrease of functional groups than 10-mGS-750 due to its high amount of functional groups.

In Fig. 3, SEM images of the carbonized materials and its EDS mapping result are displayed. Several-mm particles were observed for all the carbon materials of 0-mGS-750, 10-mGS-750 and 10-O-mGS-750. Any separation of $\mathrm{Si}$ and carbon particles was hard to be found. In Figs. 3(c) and (e), the distribution of red spots corresponding to Si metal exhibited that a few mm Si particles were homogeneously embedded within carbon matrix.

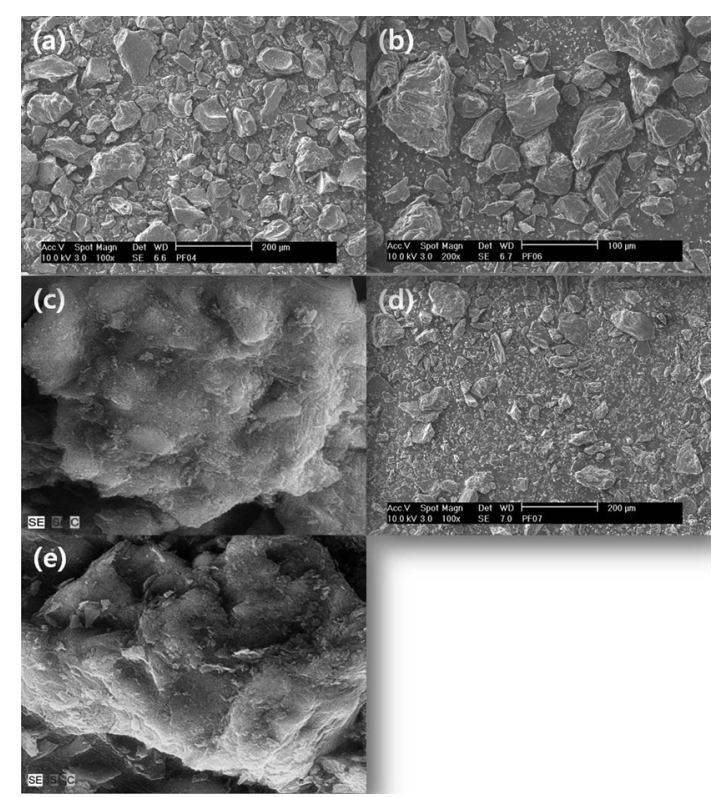

Fig. 3. SEM images; (a), (b) and (d) are 0-mGS-750, 10-mGS750 and 10-O-mGS-750, respectively. EDS mapping results; (c) and (e) are 10-mGS-750 and 10-O-mGS-750, respectively. Here, red and green color are $\mathrm{Si}$ and carbon atom, respectively. 
Fig. 4 shows XRD patterns of the carbonized composite materials. Large and broad peaks associated with carbon were observed at 27 and $43^{\circ}$, which were characteristic for carbonaceous materials prepared under $1000^{\circ} \mathrm{C} .^{2,14)}$ In addition, small peaks from Si crystal were observed at 28,47 and $56^{\circ}$. However, it was hard to find distinctive graphite peaks due to its overlapping with the carbonized product from pitch (as coke). As shown in Fig. 4(d), interestingly, the XRD patterns of 10-O-mGS-750 exhibited carbon peaks broadening near $28^{\circ}$. It was well-known that pitch becomes less graphitizable by high cross-linking in the large oxygen contents. ${ }^{17)}$ The high amount of functional groups in O-mGS can, thereby raise an similar effect and carbon of less-developed crystallinity can be observed.

In order to investigate above crystallinity change in

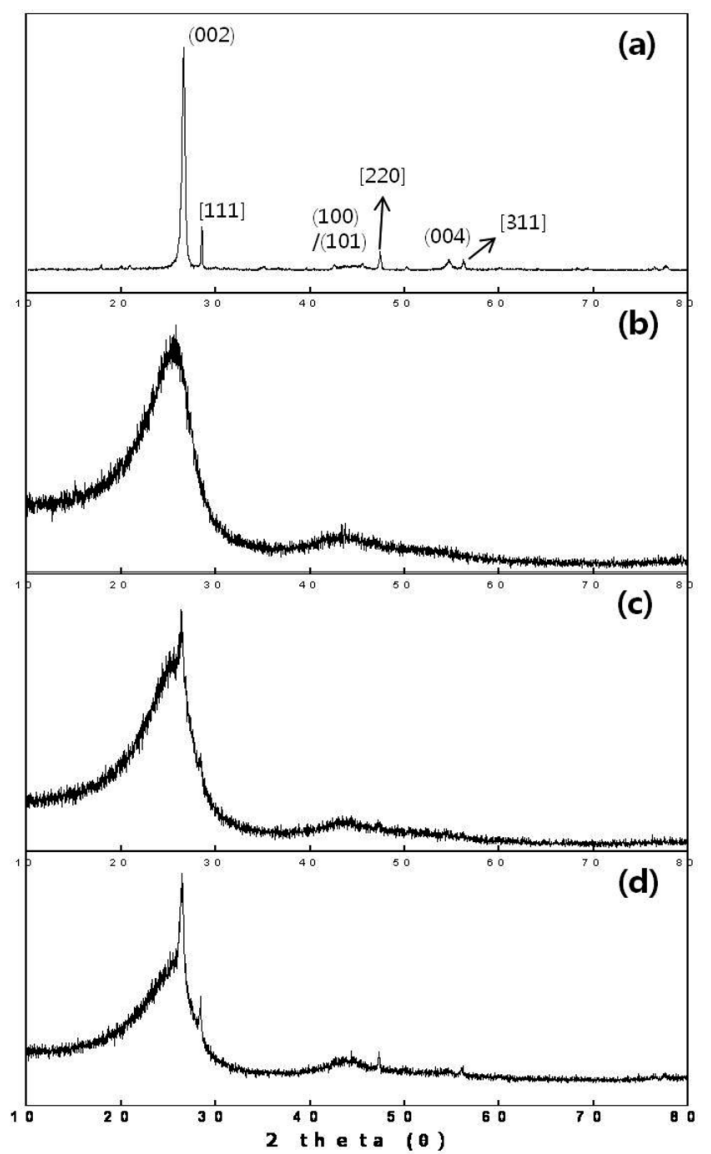

Fig. 4. XRD patterns of the prepared materials; (a), (b), (c) and (d) are mGS, 0-mGS-750, 10-mGS-750 and 10-O-mGS-750, respectively. The parenthesis and bracket indicate lattice of carbon and $\mathrm{Si}$, respectively.

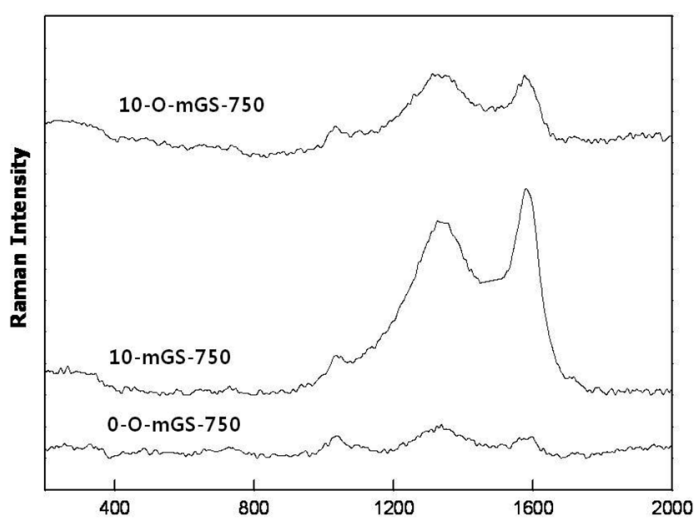

Fig. 5. Raman spectra of the prepared carbon materials; (a), (b) and (c) are 0-mGS-750, 10-mGS-750 and 10-O-mGS-750.

detail, Raman spectroscopy was carried out. In Fig. 5, Raman spectra from 300 to $2000 \mathrm{~cm}^{-1}$ are displayed. Clearly, two distinct peaks were observed near 1580 and $1360 \mathrm{~cm}^{-1}$, which corresponded to the ideal lattice vibration mode with E2g symmetry (G band) and the disordered graphite structure with A1g symmetry (D band), respectively. ${ }^{20)}$ Also, small peak near $1050 \mathrm{~cm}^{-1}$ was observed, which possibly correspond to organic components containing nitrogen functionality. ${ }^{21)}$ According to literatures, Raman vibrational modes from amorphous and crystalline Si are observed at 470 and $520 \mathrm{~cm}^{-1}$, respectively. ${ }^{22)}$ Interestingly, no peaks associated with Si were observed in Fig. 5, indicating that the embedded Si particles within carbon matrix were hard to be exposed under Raman laser beam. Similar to the Fig. 3, therefore, Si particles was completely included within carbon. From the ratio $\left(I_{\mathrm{D}} / I_{\mathrm{G}}\right)$ between $\mathrm{D}$ band $\left(I_{\mathrm{D}}\right)$ and $\mathrm{G}$ band $\left(I_{\mathrm{G}}\right)$, the crystalline size $\left(L_{\mathrm{a}}\right)$ of carbon particles can be expected empirically; $L_{\mathrm{a}}(\mathrm{nm})=4.4\left(I_{\mathrm{D}} / I_{\mathrm{G}}\right)^{-1} \cdot{ }^{23)}$ From Fig. $5, I_{\mathrm{D}} / I_{\mathrm{G}}$ values of 0 -mGS-750, 10-mGS-750 and 10-O-mGS-750 were $1.441,0.829$ and 1.002 , respectively, which reflected that crystal size of 10-mGS-750 was large and the less-developed crystallinity was observed in 10-O-mGS-750, which is coincided with the previous XRD result. ${ }^{17)}$

\subsection{Investigation of anode performance}

In order to apply our $\mathrm{Si}-\mathrm{C}$ composites into anode materials in lithium ion batteries, electrodes were fabricated and their anode performance was investigated from 0 to $2.5 \mathrm{~V}$ vs. $\mathrm{Li} / \mathrm{Li}^{+}$. In Fig. 6, the initial galvanostatic chargedischarge profiles were displayed for 0-mGS-750, 10mGS-750 and 10-O-mGS-750 electrodes under $0.1 \mathrm{C}$ rate. 

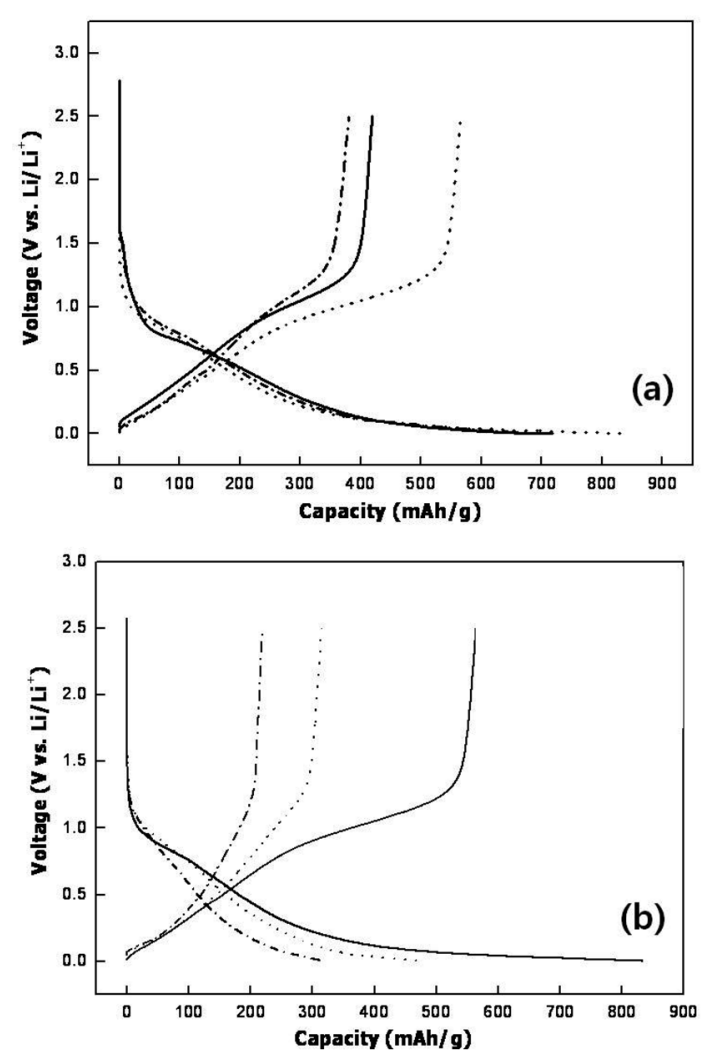

Fig. 6. Initial charge-discharge profiles mearued at $0.1 \mathrm{C}$ rate. (a); Comparison of treatment methods. 0-mGS-750, 10-mGS750 and 10-O-mGS-750 are solid, dot and dash-dot lines, respectively. (b); Heat treatment effect of 10-mGS case. 750, 850 and $1000^{\circ} \mathrm{C}$ are solid, dot and dash-dot lines, respectively.

Note that $1 \mathrm{C}$ equals to $500 \mathrm{mAh} \mathrm{g}^{-1}$ here. Interestingly, discharge capacity $\left(C_{\mathrm{dis}}\right)$ of $10-\mathrm{mGS}-750$ electrodes was as large as $565 \mathrm{mAh} \mathrm{g}^{-1}$. However, $C_{\text {dis }}$ values were 419 and $357 \mathrm{mAh} \mathrm{g}^{-1}$ for 0-GS-750 and 10-O-GS-750 electrodes, respectively. This difference of $C_{\text {dis }}$ can be relevant to Si effect in 10-GS-750 electrode and decrease of $\mathrm{Li}^{+}$storage sites in carbon caused by more disordered structure in 10-O-mGS-750. Also, an initial efficiency (IE) of $10-\mathrm{mGS}-750$ was $67.8 \%$ as listed in Table 1. In order to investigate the change of $C_{\text {dis }}$ in accordance with carbonization temperatures, anode performance of 0 -mGS-750, 850 and 1000 electrodes were analyzed and displayed in Fig. 5(b). Clearly, decrease of capacity was observed, which was attributed to the general tendency of storage sites decrease with increasing temperature ${ }^{4)}$ in the soft carbon materials. In contrast, initial efficiency remained invariant.

In Fig. 7, the rate capability was compared. As seen in
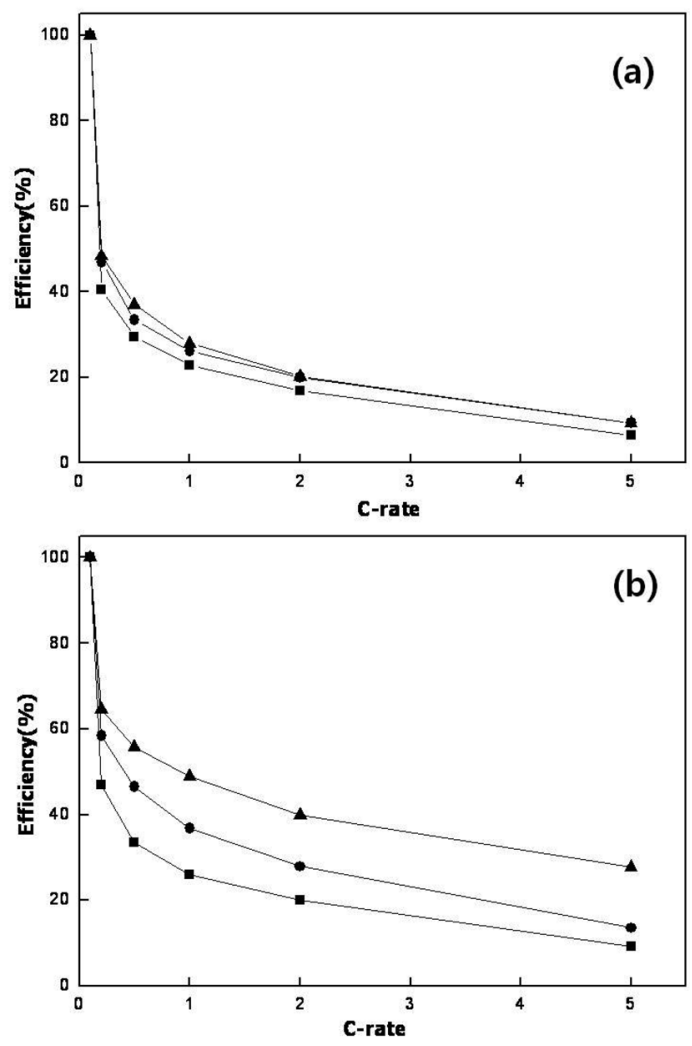

Fig. 7. Comparison of rate capability. (a); 0-mGS-750, $10-$ mGS-750 and 10-O-mGS-750 are rectangle, circle and triangle, respectively. (b); Heat treatment effect of 10-mGS case; 750, 850 and $1000^{\circ} \mathrm{C}$ are rectangle, circle and triangle, respectively.

Fig. 7(a), a similar rate capability was observed irrespective of the preparation methods. However, the rate capability became higher according to carbonization temperatures, which was probably due to an increasing crystallinity of carbons. In Fig. 8, cycleability which was measured at $0.5 \mathrm{C}$ rate until 50 cycles was shown. Note that direct comparison of $C_{\mathrm{dis}}$ values with other results in the literatures is difficult, because the electrode loading and its fabrication condition differ from those in previous studies. Only the comparison among our electrodes is therefore meaningful. A gradual decrease of capacity was observed in 0-mGS-750 electrode but 10-mGS-750 and 10-O-mGS-750 electrodes displayed a good cycle performance in spite of Si addition. From Fig. 8(b), cycleablity was similar irrespective of carbonization temperatures. In Table 2, all of anode performance results are listed for easy comparison. When the pitch and carbonization yields listed in Table 1 were considered, 

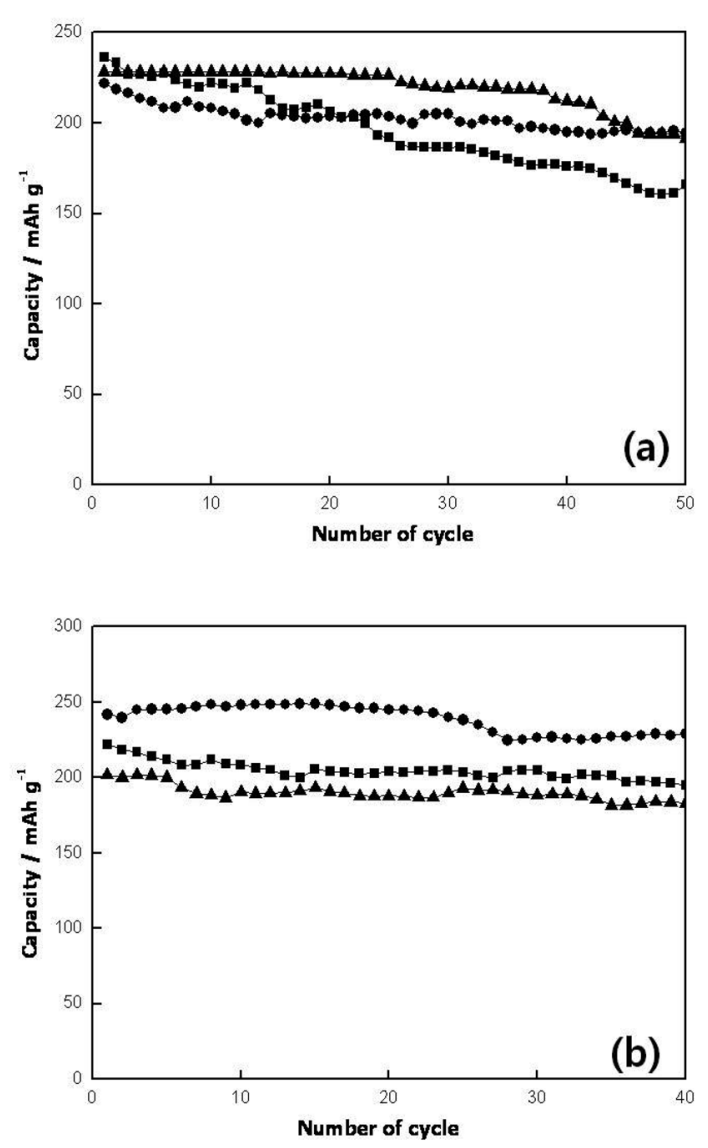

Fig. 8. Comparison of cycleablity at $0.5 \mathrm{C}$ rate. (a); 0-mGS-750, 10-mGS-750 and 10-O-mGS-750 are rectangle, circle and triangle, respectively. (b); Heat treatment effect of 10-mGS case; 750,850 and $1000^{\circ} \mathrm{C}$ are rectangle, circle and triangle, respectively.

Table 2. Anode performances of prepared carbon materials

\begin{tabular}{|c|c|c|c|c|c|}
\hline $\begin{array}{c}\text { Composite } \\
\text { material }\end{array}$ & $\begin{array}{c}\mathrm{C}_{\mathrm{ch}}{ }^{\mathrm{a}}{ }^{-1} \\
\mathrm{mAhg}^{-1}\end{array}$ & $\begin{array}{c}\mathrm{C}_{\mathrm{dis}}^{{ }^{\mathrm{b}}} \\
\mathrm{mAhg}^{-1}\end{array}$ & $\begin{array}{l}\mathrm{IE}^{\mathrm{c}} \\
\%\end{array}$ & $\begin{array}{l}\text { Cyc. }^{\mathrm{d}} \\
\%\end{array}$ & $\begin{array}{c}\text { Rate }^{\mathrm{e}} \\
\%\end{array}$ \\
\hline 0-mGS-750 & 719 & 419 & 58.3 & 74 & 23 \\
\hline 10-mGS-750 & 833 & 565 & 67.8 & 88 & 26 \\
\hline 10-mGS-850 & 514 & 357 & 69.5 & 94 & 36 \\
\hline 10-mGS-1000 & 373 & 219 & 69.0 & 91 & 49 \\
\hline 10-O-mGS-750 & 653 & 401 & 61.4 & 93 & 28 \\
\hline
\end{tabular}

anitial charging capacity $\left(C_{\mathrm{ch}}\right)$ at $0.1 \mathrm{C}$ rate.

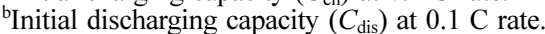

'Initial efficiency $(I E)$ at $0.1 \mathrm{C}$ rate; $I E=C_{\text {dis }} / C_{\text {ch. }}$.

${ }^{\mathrm{d}}$ Capacity retention which was obtained by dividing discharge capacity at 50 cycles by first cycle.

${ }^{\mathrm{e}}$ Rate capacity which was obtained by dividing discharge capacity at $0.5 \mathrm{C}$ rate by $0.1 \mathrm{C}$ rate.
4 5 $\mathrm{wt} \%$ Si was expected to remain after carbonization. Since the theoretical capacity of $\mathrm{Si}$ is $3580 \mathrm{mAh} \mathrm{g}^{-1}$ $\left(\mathrm{Li}_{15} \mathrm{Si}_{4}\right)$, Si particle has a capacity contribution from 140 to $180 \mathrm{mAh} \mathrm{g}^{-1}$ of active material. ${ }^{3)}$ From the literatures which have investigated carbon/Si composite anode, however, capacity was variant. ${ }^{2-4)}$ When composite between sub-mm Si powder and pitch was prepared by solvent evaporation method, the highest capacity of $550 \mathrm{mAh} \mathrm{g}^{-1}$ was observed when $5 \mathrm{wt} \% \mathrm{Si}$ was added but their cycle performance was unsatisfactory, reflecting an incomplete inclusion of Si particles within carbon. ${ }^{3)}$ From other literatures, furthermore, the reversible capacity was about $600 \mathrm{mAh} \mathrm{g}^{-1}$ even after the addition of above $10 \% \mathrm{Si}^{2,4)}$ Hence, a high capacity, an good initial efficiency and an improved cycleability were observed in our composite anode materials, which was due to the more homogeneous dispersion of Si particles within carbon.

\section{Conclusions}

From direct reaction of the milled Si-graphite or its strongly oxidized material with pitch precursor PFO, the pitch-graphite-silicon composite materials were obtained. From XRD, SEM and EDS analysis, it was observed that Si particles were homogeneously embedded within carbon particles, which was also confirmed by the peak disappearance in Raman spectra. In spite of a yield increase in the strongly oxidized graphite-silicon addition, a less-developed crystal structure was observed. From investigation of anode performance using Si-C composite materials at $750^{\circ} \mathrm{C}$, high capacity, good initial efficiency and good cycleability were observed, which was attributed to the high dispersion of Si particles within carbon. In the case of the oxidized graphite-silicon addition, capacity decrease was observed due to less crystallinity.

\section{Ackowledgement}

This work was financially supported by Korea Research Institute of Chemical Technology R\&D Project.

\section{References}

1. T. Nishida, Proceedings of the large lithium ion battery technology and application; Fourth international symposia, (1995).

2. P. Zuo, G. Yin, Z. Yang, Z. Wang and X. Cheng, Mater. Chem. Phys., 115, 757 (2009).

3. Z. Luo, D. Fan, X Liu, H. Mao,C. Yao and Z. Deng, J. 
Power Sources. 189, 16 (2009).

4. X. W. Zhang, P. K. Patil, C. Wang, Appleby, A. J. Little and F. E. Cocke, J. Power Sources. 125, 206 (2004).

5. Y.-S. Hu, R. Demir-Cakan, M.-M. Titirici, J.-O. Muller, R. Schogl, M. Antonietti and J. Maier, Angew. Chem. Int. Ed., 47, 1645 (2008).

6. H. Kim, B. Han, J. Choo and J. Cho, Angew. Chem. Int. Ed., 47, 10151 (2008).

7. A. Magasinski, B. Zdyrko, I. Kovalenko, B. Herztberg, R. Burtovyy, C. F. Huebner, T. F. Fuller, I. Luzinov and G. Yushin, App. Mater. Inter., 11, 3004 (2010).

8. M. Holzapfel, H. Buqa, W. Scheifele, P. Novak and F.-M. Petrat, Chem. Comm., 1566 (2005).

9. G. Savage, Carbon-Carbon Composites; Chapman \& Hall: London, (1993).

10. K. Kinishita, Carbon : Electrochemical and Physicochemical Properties; John Wiley \& Sons: New York, (1988).

11. H. March, Introduction to carbon science; Butterworths \& Co.: London, (1989).

12. C. S. Hahn, N. S. Cho and H. S Yang, Carbon, 19, 225 (1981).

13. S. Yoon, H. Kim and S.M. Oh, J. Power Sources, 94, 68 (2001).
14. S. Yoon, J. H. Ryu, S. M. Oh and C. W. Lee, J. NonCryst. Sol., 355, 913 (2009).

15. S. Zhao, Z. Xu, C. Xu, K. H. Chung and R. Wang, Fuel. 84, 635-645 (2005).

16. S. Yoon, S. D. Bhatt, W. Lee, S. Y. Jung and C. W Lee, Korean J. Chem. Eng., 26, 64 (2009).

17. I. Mochida, C. H. Ku and C. Y. Korai, Carbon, 39, 399 (2001).

18. J. H Choi, H. Kumagai, T. Chiba and Y. Sanada, Carbon, 33, 109 (1995).

19. Harry Marsh, Introduction to Carbon Science; Ch. 4, Butterworth \& Co. (1989).

20. A. Sadezky, H. Muckenhuber, H. Grothe, R. Niessner and U. Poschl, Carbon, 43, 1731 (2005).

21. C. Y. Panicker, H. T. Vargahese, D. Philip and H. I. S. Nogueira, Spectrochim. Acta Part A, 64, 744 (2006).

22. S. Huang, H. Xiao and S. Shou, App. Surf. Sci., 255, 4547 (2009).

23. J. M. Constantini, F. Couvreur, J. P. Salvetat and S. Bouffard, Nucl. Instr. And Meth. In Phys. Res. B, 194, 132 (2002).

24. K. Oshida and S. Bonnamy, Carbon, 402699 (2002).

25. W. Lu and D.D.L Chung, Carbon, 41, 945 (2003). 